\title{
CLASSIFICATION OF STANDARD MODULES WITH LINEAR PERIODS
}

\author{
MIYU SUZUKI
}

\begin{abstract}
Suppose that $F$ is a non-Archimedean local field of characteristic not 2 and $D$ is a central division algebra over $F$. Let $n$ be a positive integer. We show a classification modulo essentially square-integrable representations of standard modules of $\mathrm{GL}_{n}(D)$ which have non-zero linear periods. By this classification, the conjecture of Prasad and Takloo-Bighash is reduced to the case of essentially square-integrable representations.
\end{abstract}

\section{INTRODUCTION}

Suppose that $F$ is a non-Archimedean local field of characteristic not 2 and $E$ is a quadratic extension of $F$. Take a positive integer $n$ and a central division algebra $D$ over $F$ of dimension $d^{2}$. Set $G=G_{n}=\mathrm{GL}_{n}(D)$, which is the multiplicative group of $\operatorname{Mat}_{n}(D)$. Let $\nu=\nu_{n}$ denote the composition of the reduced norm of $\operatorname{Mat}_{n}(D)$ and the normalized absolute value of $F$. Assume that $E$ embeds in $\operatorname{Mat}_{n}(D)$ and let $H=C_{G}\left(E^{\times}\right)$be the centralizer of $E^{\times}$in $G$. Note that the centralizer $C_{\operatorname{Mat}_{n}(D)}(E)$ of $E$ in $\operatorname{Mat}_{n}(D)$ is a central simple algebra over $E$. Let $\chi$ be a character of $E^{\times}$and regard it as a character of $H$ by composing with the reduced norm of $C_{\operatorname{Mat}_{n}(D)}(E)$. An admissible representation $\pi$ of $G$ is said to be $(H, \chi)$-distinguished if $\operatorname{Hom}_{H}(\pi, \chi) \neq 0$. Prasad and TaklooBighash proposed a conjecture [PTB11, Conjecture 1] about $(H, \chi)$-distinguished representations. The following is $\chi=1$ case of that conjecture.

Conjecture 1.1. Let $\pi$ be an irreducible, admissible representation of $G$ with trivial central character.

(1) If $\pi$ is $H$-distinguished,

(i) The Langlands parameter of $\pi$ takes values in the symplectic group $\operatorname{Sp}_{n d}(\mathbb{C})$.

(ii) The root number satisfies $\varepsilon(\pi) \varepsilon\left(\pi \otimes \eta_{E / F}\right)=(-1)^{n} \eta_{E / F}(-1)^{n d / 2}$.

(2) If $\pi$ is an essentially square integrable representation satisfying (i) and (ii), then $\pi$ is $H$ distinguished.

Remark 1.2. The original conjecture of Prasad and Takloo-Bighash is stated under the assumption that $\pi$ corresponds to a generic representation of $\mathrm{GL}_{n d}(F)$. We will clarify the meaning of this assumption in Section 2.3. One of the new observations of this paper is that this assumption is not necessary.

This conjecture is based on the result of Tunnell [Tun83] for the case of $D=F$ with odd residual characteristic and $n=2$. There are a lot of research to generalize this result. Saito gave 
a simpler proof of Tunnell's result in [Sai93] including the case of residual characteristic 2 when the characteristic of $F$ is 0 . Prasad [Pra07] obtained a different proof of Saito's result based on a global theory.

The next three results of [PTB11], [FMW18] and [Xue] are for the case of characteristic 0. Prasad and Takloo-Bighash proved Conjecture 1.1 in [PTB11] for representations of $\mathrm{GL}_{2}(D)$ where $D$ is a quaternion algebra. They used the theta correspondence between $\mathrm{GSO}_{6}$ and $\mathrm{GSp}_{4}$. In [FMW18], Feigon, Martin and Whitehouse obtained (1) when $D=F$ and $\pi$ is supercuspidal, by using a relative trace formula. Using the same relative trace formula in a different way, Xue proved (1) for all essentially square-integrable representations and (2) for supercuspidal representations with supercuspidal Jacquet-Langlands transfer to $\mathrm{GL}_{n d}(F)$ ([Xue]). When $D$ is a quaternion algebra or $D=F$, Xue also proved (2) for all supercuspidal representations.

The following results of [Cho19] and [CM] cover the case of all non-Archimedean local fields of odd residual characteristic. In [Cho19], Chommaux proved the original conjecture (for a general character $\chi$ ) for Steinberg representations by Mackey machinery. This result also covers the case where $F$ has characteristic 0 and the residual characteristic 2. Chommaux and Matringe showed the original conjecture when $D=F$ and $\pi$ is a supercuspidal representation of depth zero ([CM]).

In [Séc20], Sécherre proved the conjecture for all supercuspidal representations when $F$ has characteristic 0 and odd residual characteristic, by using Bushnell-Kutzko type. Recently, Xue and the author proved that Conjecture 1.1 for essentially square-integrable representations reduces to the case of supercuspidal representations when the characteristic of $F$ is 0 ([SX]). See also [Séc20, Section 1.7] for a historical review on this conjecture and other related research.

The goal of this paper is to prove the next theorem.

Theorem 1.3. Suppose $P=M U$ is a standard parabolic subgroup of $G$ corresponding to the partition $n=\left(n_{1}, \ldots, n_{t}\right)$ of $n$. Let $\tilde{\pi}=\Delta_{1} \times \cdots \times \Delta_{t}$ be a standard module of $G$ induced from segments on $M$. Then, $\widetilde{\pi}$ is $H$-distinguished if and only if there is an involution $\sigma \in \mathfrak{S}_{t}$ such that $\Delta_{\sigma(i)} \simeq \Delta_{i}^{\vee}$ for all $i$ and if $\sigma(i)=i, E$ embeds in $\operatorname{Mat}_{n_{i}}(D)$ and $\Delta_{i}$ is $C_{G_{n_{i}}}\left(E^{\times}\right)$-distinguished. Here, $C_{G_{n_{i}}}\left(E^{\times}\right)$is the centralizer of $E^{\times}$in $G_{n_{i}}$.

From this, we can reduce Conjecture 1.1 (1) to the case of essentially square-integrable representations.

Theorem 1.4. Conjecture 1.1 (1) for essentially square-integrable representations implies the general case.

Combining this theorem with [Séc20] and [SX], we see that Conjecture 1.1 holds when the characteristic of $F$ is zero and the residual characteristic is different from 2. When $D$ is a quaternion algebra or $D=F$, we can remove the condition on the residual characteristic by applying [Xue] instead of [Séc20]. 


\section{Notation}

Suppose that $F$ is a non-Archimedean local field of characteristic not 2 and $E$ is a quadratic extension of $F$. Take a positive integer $n$ and a central division algebra $D$ over $F$ of dimension $d^{2}$. Set $G=G_{n}=\operatorname{GL}_{n}(D)$, which is the multiplicative group of $\operatorname{Mat}_{n}(D)$. Let $\nu=\nu_{n}$ denote the composition of the reduced norm of $\operatorname{Mat}_{n}(D)$ and the normalized absolute value of $F$. Assume that $E$ embeds in $\operatorname{Mat}_{n}(D)$ and let $H=C_{G}\left(E^{\times}\right)$be the centralizer of $E^{\times}$in $G$. Note that the centralizer $C_{\operatorname{Mat}_{n}(D)}(E)$ of $E$ in $\operatorname{Mat}_{n}(D)$ is a central simple algebra over $E$.

2.1. Parabolic subgroups. For an ordered partition $\lambda=\left(n_{1}, \ldots, n_{t}\right)$ of $n$, let $P=P_{\lambda}$ be the parabolic subgroup of $G$ consisting of matrices of the form

$$
\left(\begin{array}{ccc}
g_{1} & * & * \\
& \ddots & * \\
& & g_{t}
\end{array}\right) \in G, \quad g_{i} \in G_{n_{i}} .
$$

Write by $M=M_{\lambda}$ the Levi subgroup of $P$ consisting of matrices of the form $\operatorname{diag}\left(g_{1}, \ldots, g_{t}\right)$ with $g_{i} \in G_{n_{i}}$. Let $U=U_{\lambda}$ be the unipotent radical of $P$. Let $P_{0}$ be the minimal parabolic subgroup corresponding to the partition $\lambda_{0}=(1, \ldots, 1)$ and set $M_{0}=M_{\lambda_{0}}$. A parabolic subgroup of $G$ is called standard parabolic subgroup if it contains $P_{0}$. A standard parabolic subgroup of $G$ has a unique Levi component which contains $M_{0}$ and is called a standard Levi component. By a parabolic subgroup (resp. Levi component) we shall always mean a standard parabolic subgroup (resp. Levi component). A Levi subgroup of $G$ will be a Levi component of a parabolic subgroup of $G$. Throughout the paper, the letters $P, M$ and $U$ are reserved for parablic subgroups, their Levi components and their unipotent radicals respectively. For a Levi subgroup $M$ of $G$, we say that a parabolic subgroup of $M$ is standard if it contains $P_{0} \cap M$.

Let $P=M U$ be a parabolic subgroup of $G$ and $\mathrm{d} p$ a left Haar measure on $P$. There is a continuous character $\delta_{P}: P \rightarrow \mathbb{R}_{>0}$, called the modulus character of $P$, which satisfies

$$
\mathrm{d}\left(p p^{\prime}\right)=\delta_{P}\left(p^{\prime}\right)^{-1} \mathrm{~d} p
$$

for all $p^{\prime} \in P$.

2.2. Representations. Let $P=M U$ be a parabolic subgroup of $G$ and $\rho$ an admissible representation of $M$ on a complex vector space $V_{\rho}$. Denote by $\operatorname{Ind}_{P}^{G}\left(V_{\rho}\right)$ the space of locally constant functions $f: G \rightarrow V_{\rho}$ satisfying

$$
f(p g)=\delta_{P}^{1 / 2}(p) \rho(p) f(g)
$$

for all $p \in P$ and $g \in G$. The representation of $G$ on this space given by right translation is called the parabolically induced representation from $\left(\rho, V_{\rho}\right)$ and denoted by $\left(\operatorname{Ind}_{P}^{G}(\rho), \operatorname{Ind}_{P}^{G}\left(V_{\rho}\right)\right)$.

Let $\lambda=\left(n_{1}, \ldots, n_{t}\right)$ be the partition corresponding to $P$. Take a representation $\left(\rho_{i}, V_{i}\right)$ of $G_{n_{i}}$ for each $i$ and set $\left(\rho, V_{\rho}\right)=\left(\rho_{1} \otimes \cdots \otimes \rho_{t}, V_{1} \otimes \cdots \otimes V_{t}\right)$. This is a representation of $M$. We 
extend $\rho$ to a representation of $P$ so that $U$ acts trivially on $V_{\rho}$ and write the parabolically induced representation $\operatorname{Ind}_{P}^{G}(\rho)$ by $\rho_{1} \times \cdots \times \rho_{t}$.

Let $P^{\prime}=M^{\prime} U^{\prime}$ be a standard parabolic subgroup of $M$. We denote by $V_{\rho}\left(P^{\prime}\right)$ the subspace of $V_{\rho}$ spanned by elements of the form $\rho\left(u^{\prime}\right) v-v$ with $v \in V_{\rho}$ and $u^{\prime} \in U^{\prime}$. Set $r_{M^{\prime}, M}\left(V_{\rho}\right)=V_{\rho} / V_{\rho}\left(P^{\prime}\right)$ and we call this the Jacquet module of $V_{\rho}$ with respect to $P^{\prime}$. We write the natural projection $V_{\rho} \rightarrow r_{M^{\prime}, M}\left(V_{\rho}\right)$ by $j_{P^{\prime}}$ and define the representation $r_{M^{\prime}, M}(\rho)$ of $M^{\prime}$ on $r_{M^{\prime}, M}\left(V_{\rho}\right)$ by

$$
r_{M^{\prime}, M}(\sigma)(m) j_{P^{\prime}}(v)=\delta_{P^{\prime}}^{-1 / 2}\left(m^{\prime}\right) j_{P^{\prime}}\left(\sigma\left(m^{\prime}\right) v\right), \quad v \in V, m^{\prime} \in M^{\prime} .
$$

We briefly recall basic facts about representations of $G$. We refer to [LM16, Section 3] for details.

Let $k$ and $t$ be positive integers such that $n=t k$ and $\rho$ an irreducible supercuspidal representation of $G_{k}$. There is a positive integer $l_{\rho}$ such that the induced representation $\rho \times \nu^{l} \rho$ for $l \in \mathbb{R}$ is reducible if and only if $l \in\left\{ \pm l_{\rho}\right\}$. For a pair of integers $(a, b)$ such that $b-a=t-1$, the set

$$
[a, b]_{\rho}=\left\{\rho \nu^{a l_{\rho}}, \ldots, \rho \nu^{b l_{\rho}}\right\}
$$

is called a segment of $G$. Let $\mathfrak{L}\left([a, b]_{\rho}\right)$ denote the unique irreducible quotient of $\rho \nu^{a l_{\rho}} \times \cdots \times \rho \nu^{b l_{\rho}}$. For an irreducible essentially square-integrable representation $\pi$ of $G$, there is a unique segment $\Delta$ such that $\pi=\mathfrak{L}(\Delta)$.

Let $\Delta=[a, b]_{\rho}$ and $\Delta^{\prime}=\left[a^{\prime}, b^{\prime}\right]_{\rho^{\prime}}$ be two segments. We say that $\Delta$ and $\Delta^{\prime}$ are linked if $\Delta \cup \Delta^{\prime}$ is a segment but neither $\Delta \subset \Delta^{\prime}$ nor $\Delta^{\prime} \subset \Delta$. If $\Delta$ and $\Delta^{\prime}$ are linked and $\rho^{\prime} \nu^{a^{\prime} l_{\rho^{\prime}}}=\rho \nu^{(a+j) l_{\rho}}$ with some $j>0$, we say that $\Delta$ precedes $\Delta^{\prime}$. Let $\lambda=\left(n_{1}, \ldots, n_{t}\right)$ be a partition of $n$ and $\Delta_{i}$ a segment of $G_{n_{i}}$. Let $\mathfrak{m}$ denote the multi-set $\left\{\Delta_{1}, \ldots, \Delta_{t}\right\}$. Such a multi-set is called a multisegment. The induced representation $\Delta_{1} \times \cdots \times \Delta_{t}$ is called a standard module if for every $1 \leq i<j \leq t, \Delta_{i}$ does not precede $\Delta_{j}$. A standard module has a unique irreducible quotient $\mathfrak{L}(\mathfrak{m})$. Note that the isomorphsim class of $\mathfrak{L}(\mathfrak{m})$ depends only on $\mathfrak{m}$. For an irreducible admissible representation $\pi$ of $G$, there is a unique multisegment $\mathfrak{m}$ such that $\pi=\mathfrak{L}(\mathfrak{m})$.

A multisegment $\mathfrak{m}=\left\{\Delta_{1}, \ldots, \Delta_{t}\right\}$ is said to be totally unlinked if $\Delta_{i}$ and $\Delta_{j}$ are not linked for all $i, j=1, \ldots, t$. For a multisegment $\mathfrak{m}=\left\{\Delta_{1}, \ldots, \Delta_{t}\right\}$ of $G$, the induced representation $\Delta_{1} \times \cdots \times \Delta_{t}$ is irreducible if and only if $\mathfrak{m}$ is unlinked. If this is the case, the induced representation is independent of the ordering of the segments. When $D=F$, this is equivalent to say that $\mathfrak{L}(\mathfrak{m})$ is generic.

Let $\tilde{\pi}=\Delta_{1} \times \cdots \times \Delta_{t}$ be a standard module of $G$. For a supercuspidal representation $\rho$, set $\mathbb{Z}_{\rho}=\left\{\nu^{m l_{\rho}} \mid m \in \mathbb{Z}\right\}$ and $\pi_{\rho}=\Delta_{i_{1}} \times \cdots \times \Delta_{i_{l}}$, where $\Delta_{i_{j}} \subset \mathbb{Z}_{\rho}$ and $\Delta_{j} \not \subset \mathbb{Z}_{\rho}$ for all $j \notin\left\{i_{1}, \ldots, i_{l}\right\}$. Reordering the segments, we may write $\widetilde{\pi} \simeq \pi_{\rho_{1}} \times \cdots \pi_{\rho_{p}}$. Further we assume that $\pi_{\rho_{i}}=\left[a_{1}^{i}, b_{1}^{i}\right]_{\rho_{i}} \times \cdots \times\left[a_{q_{i}}^{i}, b_{q_{i}}^{i}\right]_{\rho_{i}}$ with $b_{1}^{i} \geq \cdots \geq b_{q_{i}}^{i}$ for each $i$. We call the expression $\pi_{\rho_{1}} \times \cdots \pi_{\rho_{p}}$ a right-ordered form of the standard module $\tilde{\pi}$.

2.3. Jacquet-Langlands transfer. In this subsection, we summarize basic facts about JacquetLanglands transfer. For details, see [Bad08, Section 2].

Jacquet-Langlands transfer is a bijection from the set of irreducible essentially square-integrable representations of $G$ to that of $\mathrm{GL}_{n d}(F)$. We write this map by JL. For an irreducible supercuspidal 
representation $\rho$ of $G_{k}$, we have $\operatorname{JL}(\rho)=\mathfrak{L}\left(\left[\frac{1-l_{\rho}}{2}, \frac{l_{\rho}-1}{2}\right]_{\rho^{\prime}}\right)$ fore some irreducible supercuspidal representation $\rho^{\prime}$ of $\mathrm{GL}_{k d / l_{\rho}}(F)$.

For a segment $\Delta=[a, b]_{\rho}$ of $G$, let $\mathrm{JL}(\Delta)$ be the segment of $\mathrm{GL}_{n d}(F)$ such that $\operatorname{JL}(\mathfrak{L}(\Delta))=$ $\mathfrak{L}(\operatorname{JL}(\Delta))$. We have

$$
\mathrm{JL}(\Delta)=\left[\frac{(2 a-1) l_{\rho}+1}{2}, \frac{(2 b+1) l_{\rho}-1}{2}\right]_{\rho^{\prime}}
$$

and it is easy to check that for two segments $\Delta$ and $\Delta^{\prime}, \Delta$ precedes $\Delta^{\prime}$ if and only if $\operatorname{JL}(\Delta)$ precedes $\operatorname{JL}\left(\Delta^{\prime}\right)$.

Let $\mathfrak{m}=\left\{\Delta_{1}, \ldots, \Delta_{t}\right\}$ be a multisegment of $G$ and $\pi=\mathfrak{L}(\mathfrak{m})$ the associated irreducible admissible representation of $G$. Define a multisegment $\operatorname{JL}(\mathfrak{m})$ of $\mathrm{GL}_{n d}(F)$ by $\mathrm{JL}(\mathfrak{m})=\left\{\mathrm{JL}\left(\Delta_{1}\right), \ldots, \operatorname{JL}\left(\Delta_{t}\right)\right\}$. We set $\operatorname{JL}(\mathfrak{L}(\mathfrak{m}))=\mathfrak{L}(\mathrm{JL}(\mathfrak{m}))$ and call it the representation of $\mathrm{GL}_{n d}(F)$ corresponding to $\mathfrak{L}(\mathfrak{m})$. In [Bad08, Section 2.7], $\operatorname{JL}(\mathfrak{L}(\mathfrak{m}))$ is denoted by $Q_{n}(\mathfrak{L}(\mathfrak{m}))$. From the above remark, we see that $\mathrm{JL}\left(\Delta_{1}\right) \times \cdots \times \mathrm{JL}\left(\Delta_{t}\right)$ is a standard module attached to $\mathrm{JL}(\mathfrak{m})$. Moreover, the corresponding representation is generic if and only if $\mathfrak{m}$ is totally unlinked.

\section{ProOF}

Let $P=M U$ be a standard parabolic subgroup of $G$ corresponding to the partition $\left(n_{1}, \ldots, n_{t}\right)$ of $n$. We recall some facts on coset representatives for $P \backslash G / H$ from [Cho19]. Let $I(P)$ be the set of symmetric matrices $s=\left(n_{i, j}\right) \in \operatorname{Mat}_{t}(\mathbb{Z})$ with non-negative entries such that the sum of $i$-th row equals $n_{i}$ for each $i=1, \ldots, t$ and when $d$ is odd, all diagonal entries are even. The set $P \backslash G / H$ is in bijection with $I(P)$ and an explicit choice of the coset representative corresponding to $s \in I(P)$, which we write by $u_{s}$, is given in [Cho19]. For a subgroup $C$ of $G$, we set $C^{u_{s}}=C \cap u_{s} H u_{s}^{-1}$. We say that $u_{s}$ or the coset represented by it is $P$-admissible if $s$ is a monomial. Take $s=\left(n_{i, j}\right) \in I(P)$ and set $u=u_{s}$. Let $P_{s}=M_{s} U_{s}$ be the standard parabolic subgroup of $G$ corresponding to the partition $\left(n_{1,1}, n_{1,2}, \ldots, n_{t, t-1}, n_{t, t}\right)$ of $n$. Note that $n_{i, j}$ could be 0 and such entries are ignored when we say $\left(n_{1,1}, n_{1,2}, \ldots, n_{t, t-1}, n_{t, t}\right)$ is a partition. Let $\widetilde{\pi}=\Delta_{1} \times \cdots \times \Delta_{t}$ be a standard module of $G$ induced from a segment $\Delta=\Delta_{1} \otimes \cdots \otimes \Delta_{t}$ on $M$.

For $s=\left(n_{i, j}\right) \in I(P)$, the Jacquet module $r_{M, M_{s}}(\Delta)$ of $\Delta$ with respect to the standarad parabolic subgroup $P_{s} \cap M=M_{s}\left(U_{s} \cap M\right)$ of $M$ is written as

$$
\Delta_{1,1} \otimes \Delta_{1,2} \otimes \cdots \otimes \Delta_{t, t-1} \otimes \Delta_{t, t},
$$

where $\Delta_{i, j}$ is a segment on $G_{n_{i, j}}$ for $i, j=1, \ldots, t$ such that $\Delta_{i}=\Delta_{i, 1} \sqcup \cdots \sqcup \Delta_{i, t}$ with the convention that $G_{n_{i}, j}=\{1\}$ and $\Delta_{i, j}$ is the trivial representation if $n_{i, j}=0$.

The following is [BM19, Proposition 5.5], which is a consequence of the Mackey theory.

Lemma 3.1. Let $\tilde{\pi}=\Delta_{1} \times \cdots \times \Delta_{t}$ be a standard module of $G$ induced from segments on $M$. Suppose that $\tilde{\pi}$ is $H$-distinguished. Then there is $s \in I(P)$ such that the Jacquet module $r_{M, M_{s}}(\Delta)$

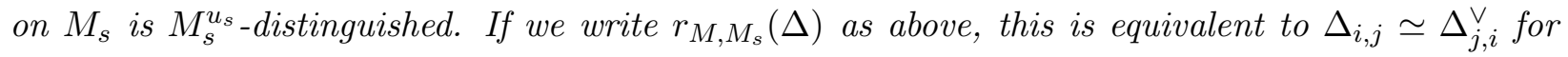
all $i, j$ and $\Delta_{i, i}$ is $C_{G_{n_{i, i}}}\left(E^{\times}\right)$-distinguished for all $i$. 
By a standard argument, we can show that only $P$-admissible cosets contribute to $H$-invariant linear forms.

Lemma 3.2. Let $\tilde{\pi}=\Delta_{1} \times \cdots \times \Delta_{t}$ be a right-ordered form of a standard module of $G$ induced from segments on $M$. If $u=u_{s}$ is not P-admissible, then the Jacquet module $r_{M, M_{s}}(\Delta)$ is not $M_{s}^{u}$-distinguished.

Proof. This result is stated in [Gur15, Lemma 3.3] for Galois involution, which is a generalization of [Mat11]. The proof holds almost verbatim for our case. We include the proof for completeness.

Assume that $u=u_{s}$ is not $P$-admissible and $r_{M, M_{s}}(\widetilde{\pi})$ is $M_{s}^{u}$-distinguished. Since $u$ is not $P$ admissible, $M_{s}$ is strictly contained in $M$. There exists $1 \leq i \leq t$ such that $\left\{n_{i, j} \mid j=1, \ldots, t\right\}-\{0\}$ is not a singleton. Let $i_{0}$ be the minimal such index and we write $\left\{j \mid n_{i_{0}, j} \neq 0\right\}=\left\{j_{1}<j_{2}<\right.$ $\left.\cdots<j_{r}\right\}$. We can write $\Delta_{i_{0}}=[a, b]_{\rho}$ with some supercuspidal representation $\rho$ and integers $a<b$. There are some integers $c$ and $d$ such that $a \leq c \leq d<b$ and $\Delta_{i_{0}, j_{1}}=[d+1, b]_{\rho}$ and $\Delta_{i_{0}, j_{2}}=[c, d]_{\rho}$. Note that we have $\Delta_{j_{1}, i_{0}} \simeq \Delta_{i_{0}, j_{1}}^{\vee} \simeq[-b,-d-1]_{\rho^{\vee}}$ and $\Delta_{j_{2}, i_{0}} \simeq \Delta_{i_{0}, j_{2}}^{\vee} \simeq[-d,-c]_{\rho^{\vee}}$. Since $\Delta=\Delta_{1} \times \cdots \times \Delta_{t}$ is a right-ordered form, $\Delta_{j_{1}} \simeq\left[a^{\prime}, b^{\prime}\right]_{\rho} \vee$ for some integers $a^{\prime} \leq-b$ and $b^{\prime} \geq-c \geq-d>-d-1$. In particular, $i_{0}>1$. Write $\Delta_{j_{1}, 1} \simeq\left[e, b^{\prime}\right]_{\rho^{\vee}}$ with some $-d \leq e \leq b^{\prime}$. Then we get $\Delta_{1, j_{1}} \simeq\left[-b^{\prime},-e\right]_{\rho}$ and since $i_{0}>1$, we have $\Delta_{1}=\Delta_{1, j_{1}}$. This contradicts the fact that $\Delta=\Delta_{1} \times \cdots \times \Delta_{t}$ is a right-ordered form since $-e<b$.

The main theorem is a straightforward consequence of Lemma 3.2.

Proof of Theorem 1.3. Suppose that $\Delta$ is $H$-distinguished. From Lemma 3.1, there is $s \in I(P)$ such that $r_{M, M_{s}}(\Delta)$ is $M_{s}^{u_{s}}$-distinguished. By Lemma 3.2, $u=u_{s}$ is $P$-admissible. In other words, $s$ is a monomial matrix in $\operatorname{Mat}_{t}\left(\mathbb{Z}_{\geq 0}\right)$. Let $\sigma \in \mathfrak{S}_{t}$ be the corresponding permutation. Then $\sigma$ is an involution and satisfies the desired conditions.

By the same argument as the proof of [MO18, Proposition 2.9], we obtain the converse implication.

Now we deduce Theorem 1.4 from Theorem 1.3.

Proof of Theorem 1.4. Let $\tilde{\pi}=\Delta_{1} \times \cdots \times \Delta_{t}$ be a standard module of $G$ which has a surjection onto $\pi$. Since $\pi$ is $H$-distinguished, so is $\tilde{\pi}$. Let $\sigma \in \mathfrak{S}_{t}$ be the permutation of Theorem 1.3. It is easy to check that the $L$-parameter of $\pi$ takes values in the symplectic group. What we have to show is the equation of Conjecture 1.1 (ii). We have

$$
\varepsilon(\pi) \varepsilon\left(\pi \otimes \eta_{E / F}\right)=\prod_{i=1}^{t} \varepsilon\left(\Delta_{i}\right) \varepsilon\left(\Delta_{i} \otimes \eta_{E / F}\right) .
$$

Suppose that $\Delta$ is $H$-distinguished. Let $\sigma \in \mathfrak{S}_{t}$ be the involution in Theorem 1.3. For each $i$, let $\omega_{i}$ be the central character of $\Delta_{i}$. Since $\Delta_{\sigma(i)} \simeq \Delta_{i}^{\vee}$, we have

$$
\varepsilon\left(\Delta_{i}\right) \varepsilon\left(\Delta_{\sigma(i)}\right)=\omega_{i}(-1), \quad \varepsilon\left(\Delta_{i} \otimes \eta_{E / F}\right) \varepsilon\left(\Delta_{\sigma(i)} \otimes \eta_{E / F}\right)=\omega_{i}(-1) \eta_{E / F}(-1)^{n_{i} d} .
$$


Hence we get

$$
\varepsilon(\pi) \varepsilon\left(\pi \otimes \eta_{E / F}\right)=\prod_{i ; \sigma(i)<i} \eta_{E / F}(-1)^{n_{i} d} \prod_{i ; \sigma(i)=i} \varepsilon\left(\Delta_{i}\right) \varepsilon\left(\Delta_{i} \otimes \eta_{E / F}\right) .
$$

Let $i$ be a fixed point of $\sigma$. Since $\Delta_{i}$ is $C_{G_{n_{i}}}\left(E^{\times}\right)$-distinguished, we have

$$
\varepsilon\left(\Delta_{i}\right) \varepsilon\left(\Delta_{i} \otimes \eta_{E / F}\right)=(-1)^{n_{i}} \eta_{E / F}(-1)^{n_{i} d / 2}
$$

from the essentially square-integrable case. Therefore the above product equals $(-1)^{n} \eta_{E / F}(-1)^{n d / 2}$.

Corollary 3.3. (1) Conjecture 1.1 holds without the condition that $\pi$ corresponds to a generic representation of $\mathrm{GL}_{n d}(F)$.

(2) For representations corresponding to generic representations, i.e. representations induced from totally unlinked multisegments, Theorem 1.3 provides a complete classification of $\mathrm{H}$ distinguished representations modulo essentially square-integrable representations.

Proof. First we consider the first assertion. In the proof of Theorem 1.4, we did not use the assumption that $\pi$ corresponds to a generic representation. Hence the first part of Conjecture 1.1 holds for any irreducible admissible representations. Note that Jacquet-Langlands transfer of essentially square integrable representations are essentially square integrable, hence generic. Therefore the assumption in the original conjecture of Prasad and Takloo-Bighash is unnecessary.

The second assertion is obvious from the fact that standard module is irreducible if and only if it is induced from totally unlinked multisegments.

Acknowledgement. I would like to thank Hang Xue for constant support and helpful discussion. In the previous version of this paper, I only treated representations induced from totally unlinked multisegments. I am very grateful to Nadir Matringe who suggested considering the general standard modules by the argument of [Gur15]. I am also grateful to Dipendra Prasad for answering many questions and Alberto Míngeuz for useful comments. I appreciate the anonymous referee for careful reading and pointing out several mistakes. This work is partially supported by JSPS Research Fellowship for Young Scientists No. 20J00434.

\section{REFERENCES}

[Bad08] A. I. Badulescue, Global Jacquet-Langlands correspondence, multiplicity one and classification of automorphic representations, Invent. Math. 172 (2008), no. 2, 383-438, DOI 10.1007/s00222-007-0104-8. MR2390289 $\uparrow 4,5$

[BM19] P. Broussous and N. Matringe, Multiplicity one for pairs of Prasad-Takloo-Bighash type, arXiv:1903.11051 (2019). $\uparrow 5$

[Cho19] M. Chommaux, Distinction of the Steinberg representation and a conjecture of Prasad and Takloo-Bighash, J. Number Theory 202 (2019), 200-219, DOI 10.1016/j.jnt.2019.01.009. MR3958071 个2, 5

[CM] M. Chommaux and N. Matringe, The split case of the PrasadTakloo-Bighash conjecture for cuspidal representations of level 0, arXiv:2004.05581v1. $\uparrow 2$ 
[FMW18] B. Feigon, K. Martin, and D. Whitehouse, Periods and nonvanishing of central L-values for GL(2n), Israel J. Math. 225 (2018), no. 1, 223-266, DOI 10.1007/s11856-018-1657-5. MR3805647 ^2

[Gur15] M. Gurevich, On a local conjecture of Jacquet, ladder representations and standard modules, Math. Zeit. 281 (2015), no. 3, 1111-1127, DOI 10.1007/s00209-015-1522-8. MR3421655 个6, 7

[LM16] E. M. Lapid and A. Mínguez, On parabolic induction on inner forms of the general linear group over a non-archimedean local field., Selecta Math. 22 (2016), no. 4, 2347-2400, DOI 10.1007/s00029-016-0281-7. MR3573961 个4

[Mat11] N. Matringe, Distinguished generic representations of GL(n) over p-adic fields, IMRN 2011 (2011), no. 1, 74-95, DOI 10.1093/imrn/rnq058. MR2755483 $\uparrow 6$

[MO18] N. Matringe and O. Offen, Gamma factors, root numbers, and distinction, Canad. J. Math. 70 (2018), no. 3, 683-701, DOI 10.4153/CJM-2017-011-6. $\uparrow 6$

[Pra07] D. Prasad, Relating invariant linear form and local epsilon factors via global methods, Duke Math. J. 138 (2007), no. 2, 233-261. MR2318284 ^2

[PTB11] D. Prasad and R. Takloo-Bighash, Bessel models for GSp(4), J. Reine Angew. Math. 2011 (2011), no. 655, 189-243, DOI 10.1515/CRELLE.2011.045. MR2806111 $\uparrow 1,2$

[Sai93] H. Saito, On Tunnells formula for characters of GL(2), Composit. Math. 85 (1993), no. 1, 99-108. MR1199206 个2

[Séc20] V. Sécherre, Représentations cuspidales de $\mathrm{GL}_{r}(D)$ distinguées par une involution intérieure., arXiv2005.0561 (2020). $\uparrow 2$

[SX] M. Suzuki and H. Xue, Intertwining periods and epsilon dichotomy for linear models, preprint. $\uparrow 2$

[Tun83] J. B. Tunnell, Local $\varepsilon$-factors and characters of GL(2), Amer. J. Math., posted on 1983, 1277-1307, DOI 10.2307/2374441. MR721997 ^1

[Xue] H. Xue, Epsilon dichotomy for linear models, preprint. $\uparrow 2$

Miyu Suzuki, Faculty of Mathematics and Physics, Institute of Science and Engineering, Kanazawa University, KaKUmamachi, KanaZaWA, IshiKaWA, 920-1192, JAPAN

E-mail address: miyu-suzuki@staff.kanazawa-u.ac.jp 